Bazyli DEGÓRSKI O.S.P.P.E.

(Roma, Angelicum)

\title{
LE NOZZE DI CANA NELL'ESEGESI DI SAN GAUDENZIO DI BRESCIA
}

L'articolo focalizza l'insegnamento di san Gaudenzio di Brescia, il vescovo vissuto tra il IV e il V secolo, un periodo di forte sviluppo dottrinale, riguardo il dono dello Spirito Santo, e le sue profonde interrelazioni con la salvezza e la vita della Chiesa, sulla base della sua esegesi del racconto di Cana di Galilea. Giacché il brano è cruciale per l'insegnamento patristico e cristiano preso nel suo insieme, si legge il pensiero di Gaudenzio in sinossi ${ }^{1}$, almeno nei passaggi salienti, con le interpretazioni dei Padri da cui egli dipendeva e dei Padri sui quali esercitò influsso, in modo da consentire non solo un'appropriata valutazione della sua originalità, ma anche un opportuno inserimento di questa figura nella più degna catena interpretativa e teologica dell'età patristica dalla quale spesso rischia di essere immotivatamente escluso.

1. Il Dies Spiritus Sancti. Secondo l'insegnamento di san Gaudenzio, la terza ed ultima tappa dell'intervento salvifico di Dio con l'umanità è iniziata ufficialmente alle nozze di Cana di Galilea. Si tratta qui di un dialogo sponsale di cui lo sposo è ora lo stesso Dio Incarnato, la sposa è la Chiesa, e il dono più prezioso dato dallo Sposo alla Sposa è il dono dello Spirito Santo.

Una delle caratteristiche degli ultimi tempi del tempo della Chiesa è quindi, il dono dello Spirito Santo. Tale periodo, perciò, può essere chiamato dies Spiritus Sancti. I pagani ne prendono parte attraverso l'iniziazione battesimale. San Gaudenzio utilizza pienamente questo simbolismo. Infatti, spiegando allegoricamente Gv 2,3, egli constata che questo versetto biblico esprime in modo chiaro la mancanza del vino dello Spirito Santo nella vita dei pagani. Le parole consummatum erat, cioè: deficerat vinum nuptiarum, non vanno riferite ai pagani, perché questo dono non era ancora stato dato loro; significano,

${ }^{1}$ La nostra esposizione del pensiero di san Gaudenzio si basa sostanzialmente sui seguenti studi fondamentali: G.M. Bruni, Teologia della storia secondo Gaudenzio di Brescia, Vicenza 1967, 27-60; G. Bruni, Pasqua primavera della storia. Teologia del tempo nei testi omiletici di Gaudenzio di Brescia (Scripta Pontificiae Facultatis Theologicae Marianum 55), Roma 2000, 37-71. 
invece, che l'Israele è venuto a mancare al primo sposalizio con il Signore. Infatti, con l'estinzione del ministero dei profeti l'Israele è stato privato del dono dello Spirito Santo e nessuno era in grado di dissetare i pagani, ma bisognava aspettare l'arrivo di Gesù Cristo, il quale, attraverso la grazia del battesimo, ha riempito i nuovi otri, gli uomini, con il nuovo vino ${ }^{2}$.

La tradizione patristica vede nel miracolo compiutosi a Cana di Galilea un doppio simbolo: da una parte, esso è figura dell'eucaristia, dall'altra, invece, del battesimo ${ }^{3}$. La simbologia del battesimo è già palesemente presente in Tcrtulliano $^{4}$; la ritroviamo più tardi, ad esempio, in Gregorio di Elvira ${ }^{5}$, in Arnobio il Giovane - il quale, però, vede nel vino di Cana non il simbolo dell'eucaristia, ma della passione di Cristo ${ }^{6}$ - in Eusebio 'Gallicano', in san Cesario di Arles ${ }^{8}$.

Gregorio di Elvira vede anche nel nuovo vino che riempie i fedeli la forza del battesimo9. Altri Padri, invece, sottolineano qui piuttosto l'azione dello Spirito Santo. Ad esempio, Origene vede nei nuovi otri l'immagine dell'uomo nuovo che deve progredire nella novità di vita, affinché possa ricevere il nuovo vino ossia la novità della grazia dello Spirito Santo ${ }^{10}$. Un simile insegnamento troviamo in Eusebio 'Gallicano'. Egli però è più preciso, perché vede nei vecchi otri i Giudei che con l'aceto dell'infedeltà hanno guastato in sé la fede nella Legge di Dio. Inoltre, fecero bere quest'aceto al Signore mentre pendeva dalla croce. I cristiani, secondo lui, sono invece gli otri nuovi che di continuo si rinnovano interiormente $\mathrm{e}$, perciò, ricevono il vino, cioè portano in loro lo Spirito Santo ${ }^{11}$. San Cromazio di Aquileia, invece, afferma che coloro che non credono e si comportano secondo la loro vecchia vita di peccato, non possono ottenere la nuova grazia dello Spirito Santo ${ }^{12}$. San Massimo di Torino parla, è vero, del battesimo, ma mettendo l'accento non sull'azione di Cristo, come fa san Gaudenzio, bensì dello Spirito Santo: il vino nuovo bisogna versarlo negli otri nuovi, ossia la grazia dello Spirito Santo va versata negli uomini nuovi, nei neofiti i quali diventano tali in forza di questa grazia ${ }^{13}$. Sant'Agostino collega gli otri nuovi e il vino nuovo con il dono dello Spirito Santo che è stato dato agli apostoli e ai primi discepoli nel giorno della Pentecoste ${ }^{14}$. Anche

${ }^{2}$ Cf. Gaudentius, Tractatus paschalis VIII 46-47, CSEL 68, 73.

3 Cf. J. Daniélou, Bibbia e liturgia, Milano 1958, 292-294.

${ }^{4}$ Cf. Tertullianus, De baptismo 9, 3-4, CCL 1, 284.

5 Cf. Gregorius Illiberitanus, In Canticum canticorum 1, 10-12, CCL 69, 173-174.

6 Cf. Arnobius Iunior, Expositiunculae in Evangelium in Iohannem 2, CCL 25 A, 272.

${ }^{7}$ Cf. Eusebius 'Gallicanus', Collectio homiliarum 5, 2, CCL 101, 58.

${ }^{8}$ Cf. Caesarius Arelatensis, Sermo 167, 1, CCL 104, 683.

${ }^{9}$ Cf. Gregorius Illiberitanus, In Canticum canticorum 1,11, CCL 69, 173.

${ }^{10} \mathrm{Cf}$. Origenes, De principiis [secundum translationem Rufini] I 3, 7, GCS 58, 22.

11 Cf. Eusebius 'Gallicanus', Collectio homiliarum 5, 3, CCL 101, 59.

${ }^{12} \mathrm{Cf}$. Chromatius Aquileiensis, Tractatus in Matthaeum 46, 5, CCL 9 A, 425.

${ }^{13}$ Cf. Maximus Taurinensis, Collectio sermonum antiqua, Sermo 28, 3, CCL 23, 109.

14 Cf. Augustinus, Sermo 267, PL 38, 1230; Idem, Sermo 272 B, in: G. Morin (ed.), Sancti 
san Girolamo unisce gli otri nuovi e il vino nuovo con la Pentecoste ${ }^{15}$. Sant'Agostino insegna, inoltre, che lo stesso dono dello Spirito Santo è stato elargito anche a tutti i fedeli di ogni tempo. Se essi diventano otri nuovi e attendono la grazia di Cristo, saranno abbondantemente riempiti dello Spirito Santo che diventerà in essi fonte d'amore, ed essi stessi diventeranno vino nuovo. Lo Spirito Santo li renderà capaci, come i martiri, di rinunciare al mondo, di sopportare ogni sacrificio per Cristo e di dargli un'eroica testimonianza ${ }^{16}$.

In generale, l'insegnamento di san Gaudenzio concorda con l'esegesi dei Padri, dal momento che lo Spirito Santo viene per la prima volta donato ai fedeli proprio durante i sacramenti dell'iniziazione cristiana, e questo Spirito Santo è stato mandato alla Chiesa da Cristo.

Questo grande cambiamento portato da Cristo, ribadisce il Vescovo di Brescia, non può essere inteso come qualcosa che è sorto quasi automaticamente (ex nihilo), perché esso è radicato nell'Antico Testamento e ne è continuazione e pienezza. Dato che il dono dello Spirito Santo, il cui simbolo è il vino, viene elargito con il battesimo, san Gaudenzio tratta anche le diverse tipologie di questo sacramento. Non ci allontaneremo dal nostro tema, quindi, se presenteremo il pensiero del Vescovo di Brescia a proposito dei diversi tipi di battesimo, a maggior ragione che egli stesso fa vari riferimenti allo Spirito Santo. Secondo san Gaudenzio, i tipi di battesimo sono costituiti da: le acque di cui si parla all'inizio della creazione, le acque del diluvio e le acque del Giordano.

2. Le acque del battesimo e lo Spirito Santo. L'acqua di Cana di Galilea che il Signore ha cambiato in vino, vivificata dall'azione dello Spirito Santo donato ai pagani nel battesimo, è la stessa acqua che vediamo all'inizio della creazione del mondo: è quell'acqua sulla quale si librava lo Spirito Santo:

„Ma è diventato tutto nuovo in modo tale che restasse l'origine dall'antico, dal momento che il vino è generato non dal nulla, ma dall'antico elemento dell'acqua" ${ }^{17}$.

Augustini Sermones post Maurinos reperti (Miscellanea Agostiniana, I), Romae 1930, 380; Idem, Sermo 299B, ed. G. Morin, op. cit., p. 518.

15 Cf. Hieronymus, Adversus Iovinianum 1, 30, PL 23, 265. Anche san Gregorio Magno, che scrive nel periodo molto più posteriore rispetto a san Gaudenzio o a san Girolamo, collega gli otri e il vino nuovo con la Pentecoste. Cf. Gregorius Magnus, Moralia in lob XXIII 11, 20, CCL 143B, 1159.

${ }^{16}$ Cf. Augustinus, Sermo 272 B, ed. G. Morin, op. cit., p. 385.

${ }^{17}$ Gaudentius, Tractatus paschalis VIII 48, CSEL 68, 73: „Sic tamen facta sunt omnia nova, ut origo maneret ex veteri, cum non ex nihilo vinum, sed ex antiquo aquae gignitur elemento", trad. C. Truzzi, Gaudenzio di Brescia. I sermoni, Roma 1996, 84-85. San Gaudenzio non tratta dettagliatamente il rapporto tra le acque dell'inizio della creazione e quelle del battesimo, ma la stessa menzione ci permette di collocarlo in quella ampia tradizione patristico-liturgica che scorge un parallelismo tra l'acqua e lo Spirito Santo della prima creazione e il battesimo durante il quale lo Spirito Santo compie una nuova creazione. Per quanto riguarda tale simbolica battesimale, cf. J. Daniélou, Bibbia e liturgia, op. cit., pp. 94-99. Questa simbolica è stata poi utilizzata dalla lex orandi come si vede dalla benedizione dell'acqua battesimale e di quella durante la veglia pasquale. 
Similmente al parallelismo che intercorre tra le acque di cui si parla all'inizio della creazione e le acque del battesimo, il Vescovo di Brescia nota il collegamento tra il diluvio e il battesimo:

„Per questo motivo nell'antico diluvio gli uomini morirono e nel battesimo rinascono" $"$.

Il parallelismo tra il diluvio e il battesimo permette a san Gaudenzio di scorgere una nuova tipologia: l'arca in cui si sono rifugiate le persone salvate dalla distruzione del diluvio è modello della Chiesa alla quale appartengono tutti coloro che professano la fede apostolica e sono uniti alla Chiesa cattoli$\mathrm{ca}^{19}$. Le acque del diluvio hanno fatto morire i peccatori ma, nello stesso tempo, si sono mostrate salvifiche per i giusti: per Noè e la sua famiglia. Anche le acque del battesimo distruggono la vecchia creazione per farne una nuova, innestata nella Chiesa che diventa la nuova arca della salvezza.

Questo simbolismo sarebbe già espresso in $1 \mathrm{Pt} 3,18$-21, ove si parla chiaramente del carattere distruttivo dell'acqua del battesimo che fa sì che il battezzato diventi partecipe del mistero della morte del Salvatore distruggendo l'uomo vecchio. Tutto ciò è conforme anche con l'insegnamento di san Paolo e la tipologia posteriore molto sviluppata dalla tradizione patristicoliturgica in armonia con la mens, con il sensus Ecclesiae ${ }^{20}$. Infatti, già Origene, appellandosi direttamente all'insegnamento di san Pictro, vedeva nel diluvio il tipo e il preannunzio del battesimo ${ }^{21}$; e, per quanto riguarda i Padri latini, anche Tertulliano scorgeva nelle acque del diluvio la figura e il preannunzio del battesimo. Anzi, lo stesso diluvio era interpretato come un peculiare genere di battesimo: una purificazione scesa su tutta la terra ${ }^{22}$. Sant'Ambrogio, contemporaneo di san Gaudenzio, constatava che il diluvio era immagine del battesimo $^{23}$, e vi dedicò un'intera trattazione ${ }^{24}$. Infatti, come il diluvio nell'Antico Testamento, così ora il battesimo lava tutti i peccati risuscitando il peccatore a vita nuova e facendo di lui un giusto ${ }^{25}$. Sant'Agostino vede nel diluvio il mistero del battesimo: come ai tempi di Noè la terra è stata purificata dalle iniquità, così grazie alle acque del battesimo vengono tolti tutti i peccati dell'uomo. L'arca, invece, costruita da un legno immarcescibile, diventa figura

${ }^{18}$ Tractatus paschalis VIII 49, CSEL 68, 73-74: ,[...] et idcirco in divino diluvio antiquo homines perierunt et nunc in baptismo renascuntur", trad. C. Truzzi, op. cit., p. 85).

19 Cf. ibidem, pp. 73-74.

${ }^{20}$ Cf. ad esempio, J. Daniélou, Déluge, baptème et jugement „Dieu Vivant” 8 (1947) 97-111; R. Beraudy, L'iniziazione cristiana, in: A.G. Martimort (ed.), La Chiesa in preghiera. Introduzione alla liturgia, Roma 1966, 601.

${ }^{21} \mathrm{Cf}$. Origenes, De principiis II 5, 3, GCS 22, 136.

${ }^{22}$ Cf. Tertullianus, De baptismo 8, 4, CCL 1, 283.

23 Cf. Ambrosius, De sacramentis I 6, 23, CSEL 73, 25.

24 Cf. ibidem II 1, 1, CSEL 73, 25.

${ }^{25}$ Cf. ibidem, p. 26. 
della Chiesa ${ }^{26}$. Questo legno che non marcisce simboleggia anche le anime dei santi e dei giusti che appartengono alla Chiesa. Come nell'arca, però, c'erano animali puri e impuri, così anche nella Chiesa ci sono persone buone ma anche cattive $^{27}$. San Massimo di Torino, contemporaneo a san Gaudenzio e proveniente dalla stessa regione, vide nel mistero del diluvio dei quaranta giorni il battesimo che purifica ${ }^{28}$ : esso, per il peccatore, è un diluvio che fa di lui un fedele. Infatti, attraverso quel lavacro purificante istituito dal Signore viene annientata l'ingiustizia e salvata dalla perdizione la giustizia ${ }^{29}$.

Un terzo tipo di battesimo, infine, per san Gaudenzio sono le acque del Giordano nelle quali il Salvatore ha ricevuto da san Giovanni il battesimo di penitenza. Immergendosi in quelle acque il Signore le ha santificate. In quel momento è sceso su di lui lo Spirito Santo in forma di colomba ${ }^{30}$. Questa tipologia che vede il battesimo nelle acque all'inizio della creazione e nelle acque del diluvio (immagini prese dall'Antico Testamento), nonché nelle acque del Giordano e nell'acqua cambiata in vino alle nozze di Cana di Galilea (immagini dal Nuovo Testamento) ${ }^{31}$, si basa sull'idea esegetica dell'unità progressiva e dinamica dei due Testamenti. Infatti, il Santo Vescovo così afferma:

„E così non pensare che sia da disprezzare la legge della lettera, dalla quale, per intervento di Gesù, i fedeli servi attingono lo Spirito Santo" ${ }^{\text {"32. }}$.

La Legge, perciò, è la base dalla quale Gesù, servendosi dei suoi servi fedeli, manda lo Spirito Santo che vivifica la lettera morta della Legge veterotestamentaria la quale, essendo priva della vita, causava soltanto morte ${ }^{33}$. L'unione dei due Testamenti è stata, inoltre, chiaramente affermata attraverso la presenza alle nozze di Cana, di Mosè, da una parte, (allegoricamente rappresentato nella persona del maestro di tavola) e di Maria, dall'altra.

In questa interpretazione, san Gaudenzio è originale. Infatti, sembra sia stato il primo a vedere nel maestro di tavola il simbolo di Mosè. Tale tipologia sarà poi ripresa da autori posteriori ${ }^{34}$. La tradizione, invece, vedeva nel mae-

${ }^{26}$ Cf. Augustinus, Sermo 260C, ed. G. Morin, op. cit., p. 334.

27 Cf. Idem, Sermo 264, PL 38, 1216.

${ }^{28}$ Cf. Maximus Taurinensis, Sermo 50, 2, CCL 23, 198.

${ }^{29} \mathrm{Cf}$. ibidem.

${ }^{30}$ Cf. Gaudentius, Tractatus paschalis II 33, CSEL 68, 32.

31 Il rito della benedizione dell'acqua durante la veglia pasquale possiede nella liturgia latina lo stesso quadruplo schema: le acque all'inizio della creazione; le acque del diluvio; l'acqua di Cana di Galilea; l'acqua del battesimo.

32 Gaudentius, Tractatus paschalis VIII 49, CSEL 68, 73: „Nec legem litterae existimes esse temnendam, unde spiritus sanctus Iesu operante per fideles ministros hauritur".

${ }^{33}$ Cf. ibidem, CSEL 68, 73-74.

${ }^{34} \mathrm{Cf}$. ad esempio: Arnobius Iunior, Expositiunculae in Euangelium in Iohannem 2, CCL 25 A, 272; Isidorus Hispalensis, Allegoriae quaedam sanctae Scripturae. Ex Novo Testamento 234, PL 83, 128. 
stro di tavola non Mosè ma san Paolo Apostolo - l'amministratore della casa del nuovo sposo, nella Chiesa di Cristo ${ }^{35}$. Un autore anonimo, inoltre, che alcuni identificano con san Girolamo, vedeva nel maestro di tavola san Giovanni il Battista, dato che egli aveva assaggiato, 'bevuto la passione' già prima di Cristo $^{36}$, e dato che il maestro di tavola negli sposalizi doveva essere un esperto conoscitore di tutte le prescrizioni, doveva essere un perito della Legge. Per questa ragione Beda il Venerabile, all'inizio del medioevo, riassumendo in un certo senso tutta la tradizione patristica a lui precedente, vede nel maestro di tavola famosi dottori della Legge: Nicodemo, Gamaliele, o anche il suo discepolo Saulo, divenuto poi san Paolo ${ }^{37}$.

3. Maria e lo Spirito Santo. San Gaudenzio insegna che proprio grazie all'intercessione di Mosè, il signore dell'antico sposalizio tra Dio e l'Israele, sappiamo che Dio non è soltanto uno, ma che si è rivelato anche come Trinità (cf. Dt 6,4). Grazie a Mosè conosciamo anche tutto ciò che è accaduto durante la creazione del mondo (cf. Gn 1,1 s.) e dell'uomo (cf. Gn 1, 27 s.), la felicità del paradiso (cf. Gn 2, 8 s.), la prevaricazione (cf. Gn 3), la generazione dei giusti e degli ingiusti (cf. Gn 5, $1 \mathrm{~s}$.), il diluvio (cf. Gn 6-8). Inoltre, è stato proprio Mosè a preannunziare ai Giudei la venuta di Cristo-profeta (cf. Dt 18, 15 s.; At 3, 22 s.). Anche a Cana di Galilea Mosè, grazie ai suoi meriti, compie l'ufficio di maestro di tavola ${ }^{38}$. Proprio a lui i servi portano il nuovo vino, egli però, meravigliato della squisitezza del dono battesimale elargito ai pagani dallo Spirito Santo, loda la generosità dello sposo che ha preparato per i pagani un vino così prezioso ${ }^{39}$.

Commentando il testo di Gv 2, 4-5, san Gaudenzio introduce un prezioso elemento mariano ${ }^{40}$ : la Madre del Salvatore è l'immagine biblica dei giusti, intercede presso l'eterno Figlio di Dio, da lei nato, per donare a noi, uomini bisognosi, le delizie del vino celeste:

„[...] così anche intendi la madre del Signore come figura del popolo dei santi patriarchi, profeti e giusti tutti, dal quale nel Vangelo si fa vedere che lo stesso Signore ha preso la nostra carne [...]. Questa madre del Signore, pertanto, ossia la generazione dei patriarchi e dei profeti ha interceduto per noi pagani presso

${ }^{35}$ Cf. Eusebius 'Gallicanus', Homiliae 5, 5, CCL 101, 60; Caesarius Arelatensis, Sermo 168, 5 , CCL 104, 690 (ambo i testi sono strettamente collegati; non è escluso che derivino dalla medesima fonte); Anonymus, Commentarium in Iohannem 2, 9, CCL 108 C, 112.

${ }^{36} \mathrm{Cf}$. Auctor Incertus [Hieronymus Stridonensis ?], Expositio Evangelionum. In Ioannem, PL 30, 578D.

${ }^{37}$ Cf. Beda Venerabilis, Homiliarum evagelii I 14, CCL 122, 103.

${ }^{38}$ Cf. Gaudentius, Tractatus paschalis IX 42-43, CSEL 68, 88 ; cf. ibidem 40-43, CSEL 68, 87-89.

39 Cf. ibidem 48, CSEL $68,90$.

40 Cf. ibidem 13-14, CSEL 68, 78-79. A proposito della presenza di Maria alle nozze di Cana, cf. A. Bresolin, L'esegesi patristica di Gv. 2, 4, Vicenza 1959. 
l'eterno Figlio di Dio e suo discendente secondo la carne, perché donasse a noi, che ne avevamo bisogno, la letizia del vino celeste" ${ }^{\text {. }}$.

Conformemente al suo metodo esegetico, san Gaudenzio presenta la Madre del Signore in una doppia dimensione: da una parte, ella appare come la genitrice di Cristo secundum carnem che intercede affinché i novelli sposi abbiano del vino; dall'altra invece, conformemente ad un'esegesi spirituale della Sacra Scrittura, Maria raffigura i patriarchi, i profeti e i giusti, diventando persino una generatio patriarcharum et prophetarum e una plebs di tutti i giusti del piano salvifico dell'Antico Testamento.

Un simile pensiero si trova in Eusebio 'Gallicano' che vede nella Madre del Signore presente a Cana di Galilea la stessa Sinagoga ${ }^{42}$; in Origene, secondo Gregorio di Elvira ${ }^{43}$, in Apponio e, infine, in san Gregorio Magno. Questi autori, però, non menzionano palesemente Maria e, quindi, non commentano lo sposalizio di Cana di Galilea. Parlano, invece, della Sinagoga come di colei che ha partorito Cristo secondo la carne e, in questo senso, identificano Maria con la Sinagoga ${ }^{44}$. Anche sant'Agostino, spiegando in quale senso l'uomo lascerà il padre e la madre e si unirà alla sua moglie (cf. Mt 19, 5; Ef 5, 31), afferma che anche Cristo ha lasciato la madre, cioè la Sinagoga dalla quale era nato secondo la carne, e si è unito con la Chiesa che egli aveva raccolto da tutte le nazioni ${ }^{45}$. La stessa spiegazione è in Prospero di Aquitania il quale cita letteralmente il testo del Vescovo di Ippona ${ }^{46}$. In un altro luogo, sant'Agostino afferma che, anche se Cristo aveva preso il corpo dalla Vergine Maria, anche la Sinagoga può essere considerata sua madre dato che apparteneva al Popolo Eletto $^{47}$. Spiegando, invece, il versetto della Madre e dei suoi fratelli che lo

${ }^{41}$ Tractatus paschalis IX 15-16, CSEL 68, 79: ,[...] ita eniam matrem domini figuraliter accipe sanctorum patriarcharum et prophetarum iustorumque omnium plebem, unde originem carnis nostrae idem dominus traxisse in evangelio perhibetur [...]. Haec ergo mater domini, generatio patriarcharum et prophetarum, intercessit pro nobis gentibus apud aeternum filium dei et suum secundum carnem natum, ut donaret nobis indigentibus caelestis vini laetitiam", trad. C. Truzzi, op. cit., p. 90; cf. ibidem 22, CSEL 68,81 .

${ }^{42}$ Cf. Eusebius 'Gallicanus', Homiliae 5, 3, CCL 101, 59.

43 Anche lo stesso Gregorio di Elvira condivide quest'opinione, cf. Gregorius Illiberitanus, In Canticum canticorum 2, 1, CCL 69, 179-180.

${ }^{44}$ Cf. idem, Tractatus Origenis de libris Sanctarum Scripturarum 7, 20, CCL 69, 60; Apponius, In Canticum canticorum expositio 5, 48, CCL 19, 138; Gregorius Magnus, Moralia in Iob 2, 36, CCL $143,257$.

${ }^{45}$ Cf. Augustinus, In Iohannis evangelium tractatus 9, 10, CCL 36, 96; idem, Contra Faustum 12, 8, CSEL 25, 337. Cf. anche ibidem, 22, 39, CSEL 25, 632.

${ }^{46}$ Cf. Prosper Aquitanus, Liber sententiarum 330, CCL 68 A, 340. Lo stesso testo, con piccole varianti, è riportato anche da uno dei discepoli di Cassiodoro, cf. Cassiodori discipulus, Commentaria in epistulas sancti Pauli. Ad Ephesios 5, PL 68, 623.

${ }^{47}$ Cf. Augustinus, Quaestiones in Heptateuchum. Quaestiones Iudicum 49, 16, CCL 33, 367; cf. anche idem, Enarrationes in Psalmos 44, 12, CCL 38, 502. 
cercano (cf. Mt 12, 46-49; Mc 3, 31-34; Lc 8, 19-21), constata che questa Madre che rimane fuori è tipo della Sinagoga ${ }^{48}$. La stessa spiegazione si trova in san Girolamo $^{49} \mathrm{e}$ in san Gregorio Magno ${ }^{50}$; e una sintesi di questo insegnamento si può ravvisare dapprima in Cassiodoro ${ }^{51}$ e nei suoi discepoli ${ }^{52} \mathrm{e}$, in seguito, nell'ultimo Padre della Chiesa latina, sant'Isidoro di Siviglia ${ }^{53}$.

Ritornando all'insegnamento di san Gaudenzio, vediamo come Maria abbia impetrato il dono dello Spirito Santo per le nazioni pagane, non soltanto come madre di Cristo secondo la carne, ma come ultima personificazione della speranza e dell'attesa dei giusti di tutti i tempi. San Gaudenzio nota che lo sposo celeste arriva alle nozze invitato dalle voci dei profeti ${ }^{54}$ che in Maria hanno trovato la loro conferma e il compimento.

Maria, quindi, è un ponte che unisce i due Testamenti. Infatti, da una parte, ella rappresenta le speranze messianiche del Popolo Eletto, dall'altra, essendo strettamente unita al Figlio, intercede affinché i pagani (il Nuovo Israele presente in lei che ha ricevuto appieno lo Spirito Santo ${ }^{55}$ ) possano gustare quel nuovo vino messianico.

La costatazione di Maria: „vinum non habent” (Gv 2,3), fa comprendere la misera situazione spirituale dei pagani che sono completamente privi della grazia dello Spirito Santo, di quella forza nuova del vino messianico ${ }^{56}$. Altri Padri, invece, interpretano la mancanza di vino con l'esaurimento, la sterilità della Sinagoga che non vuole accettare Cristo. Eusebio 'Gallicano', ad esempio, mette sulla bocca della Sinagoga le parole che Maria pronuncia a Cana di Galilea a proposito della mancanza di vino: la Sinagoga afferma che è arrivata la fine della sterile lettera della Legge. Alla Sinagoga è venuto a mancare il vino, perché essa, la vigna amata ed eletta da Dio, ha cessato di produrre uva e ha cominciato a generare soltanto cardi e spine, e perché, arrivato ormai il Signore, non si può versare il vino nuovo negli otri vecchi; bisogna gettare via il lievito della vecchia malizia ${ }^{57}$.

La risposta di Gesù: „nondum venit hora mea” (Gv 2, 4), introduce alla simbologia battesimale delle nozze di Cana. L'hora della quale parla Gesù, significa, secondo san Gaudenzio, il tempo della passione dopo la quale sol-

${ }^{48}$ Cf. idem, Enarrationes in Psalmos 127, 12, CCL, 40, 1876; idem, Contra Faustum 22, 39, CSEL 25, 633.

49 Cf. Hieronymus, Commentarii in evangelum Matthaei 2, CCL 77, 101.

so Cf. Gregorius Magnus, Homiliae in Evangelia I 3, 1, PL 76, 1086C.

51 Cf. Cassiodorus, Expositio Psalmorum 68, 9, CCL 97, 609; ibidem, 138, 13, CCL 98, 1248.

52 Cf. Cassiodori discipulus, Commentaria in epistulas sancti Pauli. Ad Ephesios 5, PL 68, 623.

53 Cf. Isidorus Hispalensis, Allegoriae quaedam sanctae Scripturae. Ex Novo Testanento 211. PL 83, 125.

${ }_{54}$ Cf. Gaudentius, Tractatus paschalis VIII 43, CSEL 68, 72.

55 Cf. idem, Tractatus paschalis IX 14, CSEL 68, 79.

$56 \mathrm{Cf}$. ibidem 2-3, CSEL 68, 75.

57 Cf. Eusebius 'Gallicanus', Homiliae 5, 3, CCL 101, 59. 
tanto sarà mandato lo Spirito Santo, quando Cristo ritornerà al Padre ${ }^{58}$. Questa interpretazione va d'accordo con l'ampia tradizione esegetica patristica ${ }^{59}$, cui ascrivere anche san Gaudenzio.

Maria, piena di Spirito Santo, comprende la risposta di Gesù e, perciò, chiede e ottiene il segno che annunziava questo evento pneumatologico. Per questa ragione, ella ordinò ai servi di fare tutto ciò che Cristo comanderà loro $^{60}$. San Gaudenzio, perciò, parlando del dono dello Spirito Santo mandato nel giorno della Pentecoste, usa l'espressione „vinum Spiritus Sancti”61, constatando che ai Giudei era venuto a mancare il vino dello Spirito Santo, pcrché i profeti avevano cessato di compiere la loro missione carismatica. Infatti, essi e la Legge dell'Antico Testamento hanno operato soltanto fino a san Giovanni il Battista (cf. Mt 11, 13) ${ }^{62}$. L'espressione vinum Spiritus Sancti, che adopera san Gaudenzio, si ispira, senza dubbio, al testo di At 2, 13.

Del „vino dello Spirito Santo” parla anche direttamente san Girolamo. Infatti, commentando il libro del profeta Zaccaria, egli afferma che il Signore ha promesso nuovamente quella gioia che ai tempi dei Maccabei riempiva il popolo giudeo che cresceva e si moltiplicava. Infatti, le nazioni pagane, divenute cristiane, possono ora nutrirsi del seme della Legge di Dio e le vergini possono bere il vino dello Spirito Santo ${ }^{63}$. Altri Padri, invece, hanno visto nel dono dello Spirito Santo anche quel nuovo vino spirituale che si è sparso sulle menti degli apostoli ${ }^{64}$. Altri Padri ancora vedevano il dono dello Spirito Santo nell'immagine di quel vino del quale parla Gesù e che si deve versare negli otri nuovi. E, così, Eusebio 'Gallicano' dice che i Giudei sono otri vecchi che con il lievito dell'infedeltà hanno guastato in loro il vino della Legge; i cristiani, invece, si rinnovano interiormente di giorno in giorno e, per questo, ricevono il vino: portano in loro lo Spirito Santo ${ }^{65}$. L'ultimo Padre della Chiesa latina,

${ }^{58}$ Cf. Gaudentius, Tractatus paschalis IX 13, CSEL 68, 78-79.

${ }^{59}$ Cf. ad esempio: Augustinus, De vera religione 16, 31, CCL 32, 206; Quodvultdeus, Sermo 1: De symbolo I 5, 25, CCL 60, 319; Fulgentius Ruspensis, Epistula 14, 41, CCL 91, 436; Anonymus, Commentarium in Iohannem 2, 5, CCL 108 C, 111; Gregorius Magnus, Registrum epistularum X 21, CCL 140A, 854. Cf. anche A. Bresolin, op. cit., p. 55. A proposito del suddetto Sermo di Quodvultdeus in cui spiega il 'Simbolo Apostolico', cf. B. Degórski, Pozaaugustyńskie komentarze patrystyczne do Symbolu Apostolskiego, in: R. Knapiński (ed.), Symbol Apostolski w nauczaniu i szluce Kościoła do Soboru Trydenckiego, Lublin 1997, 204-213.

${ }^{60}$ Cf. Gaudentius, Tractatus paschalis IX 13-14, CSEL 68, 78-79.

${ }^{61}$ Cf. ibidem, 13, CSEL 68, 78-79.

${ }^{62}$ Cf. Idem, Tractatus paschalis VIII 47, CSEL 68, 73.

${ }^{63} \mathrm{Cf}$. Hieronymus, Commentarii in prophetas minores. In Zachariam 2, 10,1-2, CCL 76 A, 838.

${ }^{64} \mathrm{Cf}$. ad esempio: Chromatius Aquileiensis, Tractatus in Matthaeum 46, 5, CCL 9 A, 425; Augustinus, Sermo 267, PL 38, 1230; Sermo 299 B, ed. G. Morin, op. cit., 518; Apponius, In Canticum canticorum expositio 7, 58, CCL 19, 179-180.

${ }^{65} \mathrm{Cf}$. Eusebius 'Gallicanus', Homiliae 5, 3, CCL 101, 59-60; cf. anche Gregorius Magnus, Moralia in lob 23, 11, 20, CCL 143 B, 1159. 
infine, sant'Isidoro di Siviglia, spiega la mancanza di vino alle nozze di Cana di Galilea con il fatto che ai Giudei è stata tolta la grazia dello Spirito Santo ed è stata data, tramitc gli apostoli, alle nazioni pagane ${ }^{66}$.

4. Il mandato apostolico e lo Spirito Santo. L'interpretazione simbolica del miracolo di Cana si estende anche ad altri elementi della narrazione evangelica, andando a proporre visioni talora inedite del brano. Anzitutto l'attenzione ai servi: infatti, secondo san Gaudenzio, i servi ai quali si rivolge Gesù alle nozze di Cana, sono gli apostoli e i loro successori. L'ordine, infatti, dato a loro di riempire le giare con acqua, significa allegoricamente il mandato apostolico; la frase, cioè, riecheggerebbe: „Andate dunque e fate miei discepoli tutti i popoli battezzandoli nel nome del Padre, del Figlio e dello Spirito Santo, insegnando loro a osservare tutte quante le cose che vi ho comandate (Mt $28,19-20)^{\prime \prime 67}$.

Ciò è, ovviamente, una corrente implicazione della sua interpretazione dell'acqua in chiave battesimale e neotestamentaria, ed è, a sua volta, gravida di intuizioni. Infatti, san Gaudenzio dimostra, attraverso questo testo, che l'istituzione del battesimo da parte di Gesù era preannunciato dagli eventi biblici che si sono compiuti alle nozze di Cana. Pare che quest'interpretazione nella patristica sia stata inaugurata proprio da lui. Dopo lui, essa appare soltanto presso pochi Padri. Ad esempio, la vediamo chiaramente in Arnobio il Giovane ${ }^{68} \mathrm{e}$, indirettamente, in un autore anonimo che vede nell'ordine di riempire le giare con l'acqua il comando di Gesù dato agli apostoli di ammaestrare tutte le nazioni. Il fatto che i servi abbiano riempito le giare fino all'orlo, viene spiegato con il fatto che la predicazione degli apostoli si è estesa su tutta la terra ${ }^{69}$. Egli non parla direttamente del battesimo, sappiamo, però, che la predicazione degli apostoli - conformemente all'ordine di Cristo (cf. Mt 28, 19) - era accompagnata dall'amministrazione del battesimo.

San Gaudenzio sottolinea anche il valore della chiamata personale degli apostoli la cui vocazione consiste nel continuo prolungamento nel mondo dell'opera salvifica del Salvatore attraverso la predicazione della parola di Dio e l'amministrazione dei sacramenti ${ }^{70}$.

${ }^{66}$ Cf. Isidorus Hispalensis, Allegoriae quaedam sanctae Scripturae. Ex Novo Testamento 234, PL 83, 128.

${ }^{67}$ Gaudentius, Tractatus paschalis IX 32, CSEL 68, 85: „Baptizate omnes gentes in nomine Patris et Filii et Spiritus Sancti, docentes eos servare omnia, quae ego mandavi vobis (Mt 28, 19 20)" ; cf. anche ibidem 27, CSEL 68, 82.

68 Cf. Arnobius Iunior, Expositiunculae in Iohannem 2, CCL 25 A 272.

${ }^{69} \mathrm{Cf}$. Anonymus, Commentarium in Iohannem 2, 2, 7, CCL $108 \mathrm{C}, 112$.

${ }^{70}$ Cf. Gaudentius, Tractalts paschalis IX 32, CSEL 68, 84, ove san Gaudenzio cita quei testi biblici che parlano dell'elezione di san Paolo (cf. $R m 1,1$ ) e degli apostoli (cf. $M t 4,19$ ) a cui Cristo aveva comandato di battezzare: „Dicatis igitur lesus ministris iniunxit opus celebrandi baptisma- 
5. La vita e lo Spirito. Cana di Galilea è immagine degli effetti salvifici del battesimo ed è una risposta esistenziale dell'umanità a così grande dono. Per questo l'acqua delle giare, che serviva alle abluzioni rituali dei Giudei e alla purificazione degli invitati alle nozze, diventa simbolo del lavacro battesimale ${ }^{71}$ che purifica tutto l'uomo. Le sei giare di pietra rappresentano i sei sensi dell'uomo (occhi, orecchi, naso, bocca, mani, piedi) che passano dallo stato di morte causato dall'idolatria alla vita, che è condizione di comunione con il vero Dio, con Cristo che è la vita e la risurrezione ${ }^{72}$.

Tale interpretazione risulta essere unica nell'antichità cristiana. Infatti, ad esempio, Origene vede nelle sei giare i sei giorni della creazione del mondo: sono sei le giare a causa di coloro che, vivendo in questo mondo, si purificano, dato che il mondo è stato creato in sei giorni ${ }^{73}$. Sant'Agostino interpreta le giare come sei periodi della storia del mondo che Cristo ha riempito con il dono del suo vino, cioè con la grazia salvifica. Il primo periodo, secondo lui, si estendeva da Adamo a Noè; il secondo, da Noè ad Abramo; il terzo, da Abramo a Davide; il quarto, da Davide alla deportazione in Babilonia; il quinto, dalla deportazione in Babilonia fino a san Giovanni il Battista; il sesto, da san Giovanni il Battista sino alla fine del mondo. Perciò, la sovranità divina di Cristo riempie tutti questi sei periodi della storia dell'universo ${ }^{74}$. Un'ulteriore ripresa di questa interpretazione si ha più tardi in Primazio di Adrumeto che senza dubbio conosceva bene le opere di sant'Agostino ${ }^{75}$, nonché in Eucherio di Lione ${ }^{76}$, in san Cesario di Arles ${ }^{77}$ e in Eusebio 'Gallicano', il quale, come si vede chiaramente, ripete esattamente il testo di san Cesario $^{78}$. Anche lo pseudo-Massimo di Torino vede nelle giare i sei periodi della storia del mondo. Egli, però, fornisce anche un'altra spiegazione. Secondo

tis". In questo Tractatus 46-47, CSEL 68,89 , san Gaudenzio si occupa della proclamazione del Vangelo e della missione apostolica presso i pagani.

${ }^{71}$ Cf. ibidem 27, CSEL 68, 82.

${ }^{72}$ Cf. ibidem 28, CSEL 68, 83.

${ }^{73}$ Cf. Origenes, De principiis IV 2, 5, GCS 22, 315. L'opinione che il numero ' 6 ' fosse perfetto si basa sull'insegnamento dei pitagorei (cf. Philo Alexandrinus, Leges allegoriae 1, 3. 15). Ciò va condiviso anche da alcuni gnostici. Per Marco Mago, ad esempio, il numero '6' è perfetto perché il nome 'I $\eta$ бoūs è composto da sei lettere (cf. Irenaeus Lugdunensis, Adversus haereses I 14, 4). La maggior parte dei Padri, invece, vedeva nella cifra ' 6 ' un'imperfezione perché essa è simbolo del mondo materiale cattivo che imprigiona l'anima. Per quanto riguarda la simbologia puramente biblica, il numero ' 6 ' cedeva il posto al numero perfetto ' 7 ' (il giorno del riposo di Dio), dato che il numero '6' richiamava il mondo materiale del peccato (cf. Clemens Alexandrinus, Stromata V 14, 93; Origenes, Commentarium in euangelium Matthaei 14, 5; cf. M. Simonetti (ed.), Origene, I principi, Torino 1968, 504-505, n. 36.

${ }_{74}$ Cf. Augustinus, In Iohannis euangelium tractatus 9, 6, CCL 36, 93-94.

75 Cf. Primasius Hadrumetinus, Commentarius in Apocalypsin 2, 5, CCL 92, 62.

${ }^{76} \mathrm{Cf}$. Eucherius Lugdunensis, Formulae spiritalis intelligentiae 11, 6, PL 50, 770.

77 Cf. Caesarius Arelatensis, Sermo 167, 1, CCL 104, 683; Sermo 168, 1, CCL 104, 688.

${ }^{78}$ Cf. Eusebius 'Gallicanus', Homiliae 5, 2, CCL 101, 57; ibidem 6, 2, CCL 101, 67. 
lui, le giare significano i cuori dei santi dottori che possiedono in loro l'acqua, cioè la dottrina della Sacra Scrittura. Il fatto, invece, che le giare siano fatte di pietra, significa i cuori dei santi: duri come la pietra che si oppongono alle tentazioni del diavolo ${ }^{79}$. Un'altra interpretazione, infine, è quella di Arnobio il Giovane che parla addirittura di sette giare di pietra e vede in esse le sette Chiese fondate sulla roccia ${ }^{80}$. Sembra che quest'interpretazione sia l'unica di questo genere nel periodo antico.

Proseguendo, san Gaudenzio afferma che la conversione che si compie mediante il battesimo, il cambiamento di vita del neofita, fa sì che l'uomo riacquisti la sua vera dignità, ottenendo nuovamente la vista per poter alzare lo sguardo al Dio vivente; nuovamente l'olfatto per essere attirato dal profumo della sua coscienza; gli orecchi per sentire le istruzioni di Dio; la bocca per proclamare, lodare e benedire il Signore; le mani per pregare Dio e i piedi per correre in chiesa ad adorare in ginocchio la Trinità ${ }^{81}$.

L'ablutio dal peccato e la conversio al Dio vivente, concepita come passaggio dall'idolatria all'amore della Trinità, dono dello Spirito Santo, è la realizzazione della nuova creazione, della vera novità. Le parole ablutio e vivificatio significano pienamente il mistero del battesimo fonte di vita nuova, principium della nuova creazione. Le acque del battesimo vivificate dallo Spirito Santo purificano dal peccato e fanno risorgere a vita nuova. Abbiamo qui a che fare con l'insegnamento di san Paolo sulla morte dell'uomo vecchio nella acque del battesimo (cf. Rm 6, 6; Ef 4, 22; 1Cor 5, 7-8; Col 3,9) e sulla vita della nuova creazione in Cristo (cf. 2Cor 5, 17; Gal 6,15).

Spiegando allegoricamente la notizia che ogni giara di pietra conteneva due e tre misure di acqua, san Gaudenzio afferma che i pagani, simboleggiati proprio dalle giare di pietra, ricevono il battesimo nel nome di tutta e indivisa la Trinità entrando in comunione con il Padre, il Figlio e lo Spirito Santo che sono simboleggiati dalle tre misure d'acqua. Infatti, nessuno può venire al Padre se non per mezzo del Figlio, e nessuno può dire che Gesù è il Signore se non per mezzo dello Spirito Santo ${ }^{82}$. Nel battesimo, quindi, così come in ogni evento salvifico, operano concordemente tutte le Persone della Trinità.

Dalla nostra ricerca risulta che il Vescovo di Brescia è stato il primo tra i Padri latini ad usare l'interpretazione allegorica che vede nelle tre misure

${ }^{79}$ Cf. Pseudo Maximus Taurinensis, Homilia 2, PL 57, 917-918.

${ }^{80} \mathrm{Cf}$. Arnobius Iunior, Expositiunculae in Euangelium in Iohannem 2, CCL 25 A, 272.

${ }^{81}$ Cf. Gaudentius, Tractatus paschalis IX 28-31, CSEL 68, 83-84. Si nota qui il forte accento che san Gaudenzio pone sulla conversio compresa come passaggio dall'idolatria, dal culto dei morti (cf. Sal 113, 12-16, citato dal Vescovo di Brescia), al culto del Dio vivente (cf. Gv 14, 19; Gv 11, 25s., citati da san Gaudenzio per sottolineare la verità che Gesù è vita e fonte di vita per tutti quelli che credono in lui).

${ }^{82}$ Cf. ibidem 33-34, CSEL 68, 84-85. San Gaudenzio si basa qui su Gv 6, 44; Gv 14, 6 e 1 Cor 12,3 . 
d'acqua delle giare la Trinità oppure i suoi doni santificatrici. La tradizione patristica posteriore attingerà diffusamente a quest'interpretazione; possiamo citare sant'Agostino ${ }^{83}$ (l'opera del Vescovo di Ippona qui citata - In Iohannis euangelium tractatus - è stata scritta dopo quest'omelia di san Gaudenzio ${ }^{84}$ ), lo pseudo Agostino ${ }^{85}$, san Cesario di Arles ${ }^{86}$, o Arnobio il Giovane che vede anche, nelle due misure d'acqua, due persone che si uniscono in matrimonio ${ }^{87}$.

Secondo san Gaudenzio, quest'unità con la Trinità invisibile alla quale partecipano i pagani grazie al sacramento dell'iniziazione, esige una risposta adeguata, e cioè tutta una vita degna del cristiano. Per questo motivo, il Vescovo di Brescia fece ogni sforzo, affinché il gregge a lui affidato - rigenerato dall'acqua del battesimo e consapevole della grazia dello Spirito Santo riversata nei cuori con quella libertà che può essere data soltanto dallo Sposo Divino $^{88}$, quel gregge che è divenuto il tempio di $\mathrm{Dio}^{89}$ - custodisse instancabilmente questo meraviglioso dono, il vino dello Spirito Santo, e progredisse conformemente alla grazia ricevuta ${ }^{90}$. Il gregge deve conservare l'innocenza che ha ricevuto ${ }^{91}$ e non cadere nella ricchezza e nell'immoralità ${ }^{92}$. Inoltre, dovrebbe fuggire l'idolatria per non diventare di nuovo povero e per non separarsi da $\mathrm{Dio}^{93}$, distinguendosi per una forte fede, speranza e carità ${ }^{94}$, conducendo una vita nello Spirito e partecipando ai carismi spirituali.

Terminando il Tractatus IX, san Gaudenzio rivolge ai fedeli l'augurio che Cristo compia nelle persone rigenerate dal battesimo anche altri segni, carismi, doni celesti che lo stesso Paraclito elargisce come vuole: la sapienza, la scienza, la fede, i carismi di guarigione e operazione di miracoli, la profezia, il dono del discernimento degli spiriti, della conoscenza di diverse lingue e della capacità di interpretarle ${ }^{95}$.

Da tutta quest'analisi risulta che nell'insegnamento di san Gaudenzio si può individuare il filo conduttore che pervade il tempo in cui è radicata. Questa storia, dal canto suo, arricchisce questo tempo di un nuovo significato, dato che

${ }^{83}$ Cf. Augustinus, In lohannis euangelium tractatus 9, 7, CCL 36, 94.

${ }^{84}$ Gli autori non sono d'accordo circa la data dell'In Iohannis euangelium tractatus. Le date proposte oscillano tra il 406 e gli anni dopo il 418, cf. A. Trapè, S. Agostino, in: A. Di Berardino (ed.), Patrologia, III, Casale Monferrato 1978, 374.

${ }_{85}$ Cf. Pseudo Augustinus, Sermones de Scripturis 91, 9, PL 39, 1922.

86 Cf. Caesarius Arelatensis, Sermo 168, 1, CCL 104, 688.

${ }^{87}$ Cf. Arnobius Iunior, Expositiunculae in Iohannem 2, CCL 25 A, 272.

88 Cf. Gaudentius, Tractatus paschalis IX 3, CSEL 68, 75.

${ }^{89}$ Cf. ibidem, 2, CSEL 68, 75; cf. anche 1Cor 3, 17.

${ }^{9)}$ Cf. idem, Tractatus paschalis VIII 50-51, CSEL 68, 74.

${ }^{11}$ Cf. idem, Tractatus paschalis IX 27, CSEL 68, 82.

92 Cf. ibidem, 2, CSEL 68, 75.

${ }^{43}$ Cf. ibidem, 2-3, CSEL 68, 75.

${ }^{94} \mathrm{Cf}$, ibidem, 34-36, CSEL 68, 85-86.

95 Cf. ibidem, 51, CSEL 68, 91. Poco dopo, san Gaudenzio cita 1Cor 12, 8-11. 
esso diventa un ininterrotto dialogo sponsale tra Dio e l'umanità: il dialogo incominciato all'inizio del tempo e sempre vivo nonostante la caduta del primo uomo, perché in ogni tempo è basato su quella magnifica testimonianza data da tanti uomini eccellenti (i giusti del periodo del primus dies; l'Israele, durante il secundus dies). Da questa schiera di giusti è uscito il Cristo-sposo che durante la consummatio saeculi, grazic al sacramento dell'iniziazione battesimale e del dono del Consolatore, ha donato ai pagani la gioiosa partecipazione al dialogo sponsale con se stesso e, attraverso di sé, con l'eterno Padre.

Il terzo periodo è il tempo della Chiesa, madre e sposa dello Spirito Santo. Questa Chiesa ha come compito di proclamare in tutto il mondo la parola di $\mathrm{Dio}^{96}$ e di amministrare il battesimo. La Chiesa-sposa è stata istituita per essere la luce tra i pagani e, in tal modo, portare la salvezza a tutti ${ }^{97}$. In questa Chiesa trovano il loro compimento e prolungamento l'opera della creazione e gli eventi biblici collegati con il diluvio e con l'arca. Infatti, tutti questi eventi si realizzano nel sacramento del battesimo che ci libera dalla prigionia del peccato e ci innesta nella comunione sponsale con il Padre nel Cristo attraverso la luce trasfigurante e vivificante dello Spirito Santo.

\section{$* * *$}

Nella pienezza dei tempi, Cristo è presente alle nozze di Cana, denominata possessio gentium, terra della gentilità. Attorno allo Sposo sono Mosè, prefigurato dal maestro di tavola, Maria che riassume e sintetizza in sé i patriarchi, i profeti e tutti i giusti dell'Antico Testamento, gli apostoli a cui verrà prefigurata la missione di predicatori della parola e di ministri del battesimo. In questo ambito, Cristo fa della Chiesa, radunata dalla moltitudine delle genti, la sua sposa, elargendo ad essa, per intercessione di Maria, il dono del nuovo vino: lo Spirito Santo. Nel Cristo-sposo confluiscono le attese degli antichi giusti prefigurati da Maria, che intercede affinché lo Sposo conceda alle genti assetate, simboleggiate da Cana città della gentilità, il dono dell'unione nuziale: lo Spirito Santo. L'ordine dato agli inservienti di riempire le giare di pietra con l'acqua, sta a significare allegoricamente la vocazione apostolica del battesimo e dell'ammaestramento. Spogliando il pensiero di san Gaudenzio da un'esegesi talora forzata, rimane valida la sua impostazione che vede nello Sposo confluire l'antica alleanza e l'inizio di un'era di sponsalità, l'epoca dello Spirito Santo, il dies lucis. L'esistenza cristiana, rinnovata dalle acque battesimali, comporta di custodire integro il vino dello Spirito, l'innocenza donata, non ricadendo nell'idolatria per non essere dispersi da Dio. In sintesi, si tratta per san Gaudenzio di vivere nella fede, speranza c carità, in fedeltà ai carismi riccvuti. Il suo

${ }^{96}$ Cf. ibidem, 46-47, CSEL 68, 89-90.

${ }^{47}$ Cf. ibidem, 43, CSEL 68, 88-89. San Gaudenzio cita qui At 13, 46-47 (cf. anche Is 49, 6). 
ricco e coerente insegnamento lo rivela come parte integrante della tradizione csegetica patristica, da cui trae alcuni elementi interpretativi ma a cui anche dona originali spunti che radicalizzano l'ermeneutica, peraltro inesauribile, del racconto di Cana e ne svelano la coesione pneumatologica, sacramentale ed ecclesiologica, nonché mariana e morale.

\title{
GODY W KANIE GALILEJSKIEJ W EGZEGEZIE SW. GAUDENTEGO Z BRESCII
}

\author{
(Streszczenie)
}

Artykuł uwypukla nauczanie św. Gaudentego z Brescii odnośnie do boskości Ducha Świętego na podstawie egzegezy biblijnej Godów w Kanie Galilejskiej. Zważywszy, iż perykopa ta była chętnie wyjaśniana przez Ojców Kościoła, artykuł stara się także porównać egzegezę Biskupa Brescii z egzegezą innych Ojców czy pisarzy kościelnych, aby w ten sposób jeszcze wyraźniej ukazać wartość nauczania św. Gaudentego.

Według Biskupa Brescii, gdy nadeszła pełnia czasów, Chrystus pojawil się na weselu w Kanie Galilejskiej, którą to Kanę św. Gaudenty nazywa possessio gentium, ziemią pogan. Wokól pana młodego znajdują się: Mojżesz, którego symbolizuje gospodarz weselny; Maryja, która streszcza i zawiera w sobie patriarchów, proroków i wszystkich sprawiedliwych Starego Testamentu; apostołowie, którym zostanie zlecone posłannictwo głoszenia Ewangelii i szafowania chrztu.

W takim to właśnie otoczeniu Chrystus czyni z Kościoła zgromadzonego z narodów pogańskich swoją oblubienicę, udzielając mu za wstawiennictwem Maryi daru nowego wina, którym jest Duch Święty. W Chrystusie-Oblubieńcu znajdują swe wypełnienie oczekiwania sprawiedliwych Starego Testamentu, których figurą jest Maryja, która wstawia się, aby Oblubieniec udzielił spragnionym narodom pogańskim, których symbolem jest Kana - miasto pogan - daru zaślubin małzeńskich, którym jest Duch Święty. Rozkaz dany sługom, aby napelnili kamienne stągwie wodą, symbolizuje powołanie apostołów do udzielania chrztu i do nauczania Dobrej Nowiny.

Usuwając z nauczania św. Gaudentego niektóre składniki nieco może niekiedy przesadzonej egzegezy alegorycznej, należy stwierdzić, iż egzegeza ta jest szczególnie oryginalna i cenna $z$ tego powodu, iż widzi w ewangelicznym Oblubieńcu postać, która jednoczy Stary i Nowy Testament i zapoczątkowuje okres zaślubin w dziejach świata i Kościoła, okres Ducha Świętego - jedyny i niepowtarzalny dies lucis. 